\title{
ON THE EQUICONVERGENCE BETWEEN TWO NOERLUND TRANSFORMATIONS
}

SHIMSHON ZIMERING

I. Introduction. Let $\sum u_{k}$ be a series of complex terms, with partial sum

$$
s_{n}=\sum_{k=0}^{n} u_{k}, \quad n=0,1,2, \cdots
$$

By equiconvergence of two transforms of series $t_{n}=\sum_{k=0}^{n} a_{n, k} s_{k}$ and $\rho_{n}=\sum_{k=0}^{n} b_{n, k} s_{k}$ we mean that $\lim _{n=\infty}\left\{t_{n}-\rho_{n}\right\}=0$ (see $\left.[1, \mathrm{p} .97]\right)$.

In this paper we study the equiconvergence between two Noerlund means (Theorem 1). We then give an application of this theorem to the equiconvergence of Cesàro, continuous and discontinuous Riesz transforms of the same order $r>0$, i.e. for

$$
\begin{aligned}
C_{r}\left(s_{n}\right) & =\left(\sum_{k=0}^{n}\left(\begin{array}{c}
n-k+r \\
n-k
\end{array}\right) u_{k}\right) /\left(\begin{array}{c}
n+r \\
n
\end{array}\right), \\
R_{x}^{r}\left(s_{n}\right) & =x^{-r} \sum_{k \leq x}(x-k)^{r} u_{k}
\end{aligned}
$$

and $R_{r}\left(s_{n}\right)=R_{[x]}^{r}\left(s_{n}\right)$. ([x] denotes the greatest integer which is less than $x$.)

II. A theorem of equiconvergence between two positive and regular Noerlund transformations.

Theorem 1. Let

$$
t^{(p)}\left(s_{n}\right)=\left(\sum_{k=0}^{n} p_{n-k} s_{k}\right) / \sum_{k=0}^{n} p_{k}=\left(\sum_{k=0}^{n} p_{n-k} s_{k}\right) / P_{n}
$$

and

$$
\begin{aligned}
t^{(q)}\left(s_{n}\right)=\left(\sum_{k=0}^{n} q_{n-k} s_{k}\right) / \sum_{k=0}^{n} q_{k}=\left(\sum_{k=0}^{n} q_{n-k} s_{k}\right) / Q_{n}, & \\
n & =0,1,2, \cdots,
\end{aligned}
$$

be two positive and regular Noerlund transformations, i.e.

$$
\begin{array}{lll}
p_{0}>0, p_{n} \geqq 0, & n=1,2, \cdots ; & \lim _{n=\infty} p_{n} / P_{n}=0, \\
q_{0}>0, q_{n} \geqq 0, & n=1,2, \cdots ; & \lim _{n=\infty} q_{n} / Q_{n}=0,
\end{array}
$$

Received by the editors March 10, 1966. 
(see $[2, p .64])$.

Suppose that

$$
\lim _{n=\infty} Q_{n} / P_{n}=\alpha \quad(0<\alpha<\infty)
$$

and that the sequence $\left\{d_{n}\right\}$, which is defined by

(3) $\sum_{n=0}^{\infty} d_{n} Z^{n}=\left\{\alpha-\left(\sum_{n=0}^{\infty} Q_{n} Z^{n}\right) /\left(\sum_{n=0}^{\infty} P_{n} Z^{n}\right)\right\} /(1-Z),|Z|<1$, satisfies

$$
\sum_{n=0}^{\infty}\left|d_{n}\right|<\infty, \quad \sum_{n=0}^{\infty} d_{n} Z^{n} \neq 0, \quad|Z| \leqq 1,
$$

and

$$
\limsup _{n=\infty}\left(\left|\frac{Q_{n}}{P_{n}}-\alpha\right| \frac{\sum_{0}^{n} Q_{k}}{Q_{n}}\right)<\left|\sum_{0}^{\infty} d_{n}\right| .
$$

Then the necessary and sufficient condition for the equiconvergence of $t^{(p)}\left(s_{n}\right)$ and $t^{(q)}\left(s_{n}\right)$ is that the sequence $\left\{u_{n}\right\}$ be summable to zero by the first transformation, i.e.

$$
\lim _{n=\infty} t^{(p)}\left(u_{n}\right)=0 \Leftrightarrow \lim _{n=\infty}\left\{t^{(p)}\left(s_{n}\right)-t^{(q)}\left(s_{n}\right)\right\}=0 .
$$

Proof of Theorem 1 . We set

$$
\begin{aligned}
\Delta_{n} & =t^{(p)}\left(s_{n}\right)-t^{(q)}\left(s_{n}\right), \quad n=0,1,2, \cdots, \\
N_{n}^{(p)} & =\sum_{n=0}^{n} p_{n-k} s_{k}, N_{n}^{(q)}=\sum_{k=0}^{n} q_{n-k} s_{k}, \quad n=0,1,2, \cdots, \\
\Delta N_{0}^{(p)} & =N_{0}^{(p)}, \Delta N_{n}^{(p)}=N_{n}^{(p)}-N_{n-1}^{(p)}, \quad n=1,2, \cdots
\end{aligned}
$$

and

$$
\rho_{n}=\left(\Delta N_{n}^{(p)}\right) / Q_{n}, \quad n=1,2, \cdots .
$$

The multiplication of equation (3) by $(1-Z) \sum_{n=0}^{\infty} N_{n}^{(p)} Z^{n}$ yields

$$
\sum_{k=0}^{n} d_{n-k} \Delta N_{k}^{(p)}=\alpha N_{n}^{(p)}-N_{n}^{(q)}, \quad n=0,1,2, \cdots ;
$$

and consequently 


$$
\begin{aligned}
\Delta_{n} & =\frac{1}{Q_{n}}\left\{\sum_{k=0}^{n} d_{n-k} \Delta N_{k}^{(p)}-\left(\frac{Q_{n}}{P_{n}}-\alpha\right) \sum_{k=0}^{n} \Delta N_{k}^{(p)}\right\} \\
& =\sum_{k=0}^{n}\left\{a_{n, k}+d_{n-k}\right\} \rho_{k}, \quad n=0,1,2, \cdots,
\end{aligned}
$$

where

(6)

$$
a_{n, k}=\left(\frac{Q_{n}}{P_{n}}-\alpha\right) \frac{Q_{k}}{Q_{n}}+\left(1-\frac{Q_{k}}{Q_{n}}\right) d_{n-k}, \quad 0 \leqq k \leqq n .
$$

As

$$
\Delta N_{n}^{(p)}=\sum_{k=0}^{n} p_{n-k} u_{k}, \quad n=0,1,2, \cdots,
$$

it follows by using (2) that

$$
\lim _{n=\infty} \rho_{n}=0 \Leftrightarrow \lim _{n=\infty} t_{n}^{(p)}\left(u_{n}\right)=0,
$$

so that it is enough to show that

$$
\lim _{n=\infty} \rho_{n}=0 \Leftrightarrow \lim _{n=\infty} \Delta_{n}=0
$$

The assertion (7) follows from the following theorem [3, Theorem 1]:

A matrix $\left\{a_{n, k}+d_{n-k}\right\}$ defines a mercerian transform for the limit zero, i.e.

$$
\rho_{n} \rightarrow 0 \Leftrightarrow \sum_{k=0}^{n}\left\{a_{n, k}+d_{n-k}\right\} \rho_{k} \rightarrow 0, \quad n \rightarrow \infty,
$$

if (4) is satisfied and if

$$
\lim _{n=\infty} \sum_{k=0}^{n}\left|a_{n, k+1}-a_{n, k}\right|=0
$$

and

$$
\underset{n=\infty}{\lim \sup _{n=0}} \sum_{k=0}^{n}\left|a_{n, k}\right|<\left|\sum_{0}^{\infty} d_{n}\right|
$$

In fact, (1) and (4) imply

$$
\lim _{n=\infty} \sum_{k=0}^{n}\left(1-\frac{Q_{k}}{Q_{n}}\right)\left|d_{n-k}\right|=0 .
$$


Consequently, it follows by (2) and (5) that the matrix $\left\{a_{n, k}\right\}$ defined by (6) satisfies the conditions (8) and (9).

III. The necessary and sufficient condition for the equiconvergence of Cesàro and Riesz transforms. We set

$$
P_{n}^{(r)}=\left(\begin{array}{c}
n+r \\
n
\end{array}\right) \text { and } Q_{n}^{(r)}=n^{r}, \quad n=0,1,2, \cdots,
$$

i.e. $t^{(p)}\left(s_{n}\right)=C_{r}\left(s_{n}\right)$ and $t^{(q)}\left(s_{n}\right)=R_{r}\left(s_{n}\right)$, where $r$ denotes a real positive number. It is obvious that (2) is satisfied with $\alpha=\Gamma(r+1)$. The sequence $\left\{d_{n}\right\}=\left\{d_{n}^{(r)}\right\}$ is defined in this case by

$$
\sum_{n=0}^{\infty} d_{n}^{(r)} Z^{n}=\frac{\Gamma(r+1)-(1-Z)^{r+1} \sum_{n=1}^{\infty} n^{r} Z^{n}}{1-Z},|Z|<1
$$

(see equation (3)). This sequence satisfies the condition (4) with

$$
\sum_{n=0}^{\infty} d_{n}^{(r)}=\frac{1}{2} \Gamma(r+2)
$$

(see [3, Lemma 1, equation 21 and Lemma 2]). Now

$$
\begin{aligned}
\left|\frac{Q_{n}^{(r)}}{P_{n}^{(r)}}-\alpha\right| \cdot \sum_{k=0}^{n} Q_{k}^{(r)} / Q_{n}^{(r)} & =\left(\Gamma(r+1)-\frac{n_{r}}{\left(\begin{array}{c}
n+r \\
n
\end{array}\right)}\right) \sum_{k=0}^{n} k^{r} / n^{r} \\
& =\frac{r}{2} \Gamma(r+1)+o(1), \quad n \rightarrow \infty
\end{aligned}
$$

this shows, by using (10), that the condition (5) is also satisfied for every $r>0$. Hence, the transformations $C_{r}\left(s_{n}\right)$ and $R_{r}\left(s_{n}\right)$ satisfy the conditions of Theorem 1. Consequently we obtain the following result:

Let $r>0$ be a real number. Then $C_{r}\left(s_{n}\right)$ and $R_{r}\left(s_{n}\right)$ are equiconvergent if and only if the sequence $\left\{s_{n}\right\}$ is Cesàro summable of order $r$ to zero, i.e.,

$$
\lim _{n=\infty} C_{r}\left(u_{n}\right)=0 \Leftrightarrow \lim _{n=\infty}\left\{C_{r}\left(s_{n}\right)-R_{r}\left(s_{n}\right)\right\}=0
$$

(see [3, Theorem 2]).

In a similar way we prove that 


$$
\begin{aligned}
\lim _{n=\infty} C_{r}\left(u_{n}\right)=0 & \Leftrightarrow \lim _{x=\infty}\left\{C_{[x]}^{r}\left(s_{n}\right)-R_{x}^{r}\left(s_{n}\right)\right\}=0 \\
& \Leftrightarrow \lim _{x=\infty}\left\{R_{[x]}^{r}\left(s_{n}\right)-R_{x}^{r}\left(s_{n}\right)\right\}=0
\end{aligned}
$$

(see $[3$, p. 84]).

The results (11) and (12) contain those of M. Riesz (see E. W. Hobson [4 p. 96], R. G. Cooke [1, p. 108 and p. 112], [5] and [6], R. P. Agnew [7] and G. M. Ortner [8]), who have given sufficient conditions for the equiconvergence of two transformations among $C_{r}\left(s_{n}\right), R_{x}^{r}\left(s_{n}\right)$ and $R_{r}\left(s_{n}\right)$.

\section{REFERENCES}

1. R. G. Cooke, Infinite matrices and sequence spaces, Macmillan, New York, 1950.

2. G. H. Hardy, Divergent series, Oxford Univ. Press, New York, 1949.

3. S. Zimering, On a Mercerian theorem and its application to the equiconvergence of Cesdro and Riesz transforms, Publ. Inst. Math. (Beograd) 1(15) (1961), 83-91.

4. E. W. Hobson, The theory of functions of a real variable, Vol. II, 2nd ed., Cambridge Univ. Press, New York, 1926.

5. R. G. Cooke, On mutual and regular T-limits, Proc. London Math. Soc. (2) 41 (1936), 114, 117-119.

6. - An extension of some recent results on mutual consistency of regular $T$ limits, J. London Math. Soc. 12 (1937), 98-105.

7. R. P. Agnew, Equiconvergence of Cesdro and Riesz transforms of series, Duke Math. J. 22 (1955), 451-460.

8. G. M. Ortner, Equiconvergence of Riesz and Cesdro transforms of series, Dissertation abstracts 26 (1963), 1076.

Battelle Institute, Geneva, Switzerland 